\title{
Neoadjuvant chemotherapy in advanced gastric cancer with non- curative factors: a Phase II study with 5-fluorouracil, leucovorin, and cisplatin
}

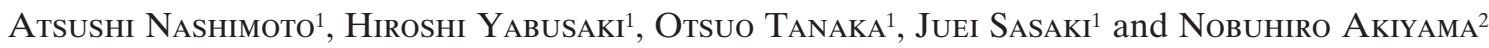 \\ ${ }^{1}$ Division of Surgery, Niigata Cancer Center Hospital, 2-15-3 Kawagishi-cho, Niigata 951-8566, Japan \\ ${ }^{2}$ Division of Internal Medicine, Niigata Cancer Center Hospital, Niigata, Japan
}

\begin{abstract}
:
Background. Neoadjuvant chemotherapy (NAC) has recently received increasing attention in an attempt to increase the rate of complete tumor resections, reduce systemic metastases, and prolong survival in patients with advanced gastric cancer. Methods. Since 1993, 21 patients with unresectable or noncurative resectable gastric cancer received NAC, consisting of 5-fluorouracil, leucovorin, and cisplatin (FLP) with at least two cycles before surgery.

Results. All except 2 patients underwent surgical treatment, and resection was performed in $18(85.7 \%)$. There were no deaths and no major morbidity following operation. There was no complete response (CR), but 12 patients $(57.1 \%)$ had a partial response (PR), the response rate was $47.6 \%$ for the primary region, $64.7 \%$ for abdominal para-aortic (No.16) lymph node metastasis, $\mathbf{4 0 . 0} \%$ for liver metastasis, and $\mathbf{1 1 . 1} \%$ for peritoneal dissemination. One-year survival of the 21 patients was $40.5 \%$, and median survival time (MST) was 322 days. MST in the responders was 571 days, and that in nonresponders was 199 days $(P<0.01)$. MST was 835 days in patients who underwent curative resection and 310 days in those who underwent non-curative surgery $(P<0.01)$. There was no grade 4 toxicity, but grade 3 leukopenia occurred in 4 patients $(19.0 \%)$, grade 3 anemia occurred in 3 patients $(14.3 \%)$, and grade 3 stomatitis in 2 patients $(9.5 \%)$. There were no serious renal disorders and no treatment-related death.

Conclusions. The combination of FLP for NAC was feasible and useful for tumor reduction, especially for No.16 lymph node metastasis. There was a survival benefit in patients whose tumor had PR or who had had curative resection. We should confirm the effect and survival benefit of FLP for NAC by a prospectively randomized clinical controlled study.
\end{abstract}

Offprint requests to: A. Nashimoto

Received for publication on Jan. 19, 1999; accepted on March 15,1999

This study was supported in part by Grants-in-Aid for Cancer Research (8S-1) from the Ministry of Health and Welfare
Key words: neoadjuvant chemotherapy, advanced gastric cancer, abdominal para-aortic lymph node metastasis, survival, response rate

\section{Introduction}

Although there has been recent progress in surgical procedures for advanced gastric cancer, the effects of extensive resection and systematic lymphadenectomy have reached their limits in survival benefit [1], and gastric cancer remains a leading cause of death in Japan. An analysis of prognostic factors clearly shows that the parameters associated with poor survival in patients with gastric cancer are distant metastases, lymph node metastases, and incomplete surgical resection [2-5]. Advanced gastric cancer with non-curative factors is a systemic disease. The current efforts to improve survival in patients with advanced gastric cancer aim to increase the rate of complete tumor resection and treat residual disease after incomplete tumor removal. Recent chemotherapy with high response rate [6-9] allows for a chance of performing radical surgery for primarily unresectable disease. High morbidity after radical surgery hinders early administration of adjuvant chemotherapy, suggesting theoretic considerations that support the use of chemotherapy prior to surgical resection [10]. Because of scarring and altered blood supply after operation drugs delivered systemically may not reach the residual tumor in sufficiently high concentrations. The premise of preoperative chemotherapy in patients with advanced gastric cancers is that it should enhance the excision rate, reduce systemic metastatic foci and primary lesions, downstage advanced cancer, reduce the viability of cancer cells (and prevent their spread during surgery), increase the in-vivo sensitivity rate in the cancer tissue, and thus prolong survival. Furthermore, more intensive chemotherapy is possible 
before than after surgery. Considering the poor prognosis of advanced gastric cancer with non-curative clinical factors, neoadjuvant chemotherapy (NAC) was employed to prolong survival and improve the quality of their remaining life in these patients.

\section{Patients and methods}

From January 1993 to May 1997, 21 consecutive patients with unresectable or non-curative resectable gastric cancer were entered into this phase II trial if they fulfilled the following eligibility criteria: (1) histologically confirmed adenocarcinoma of the stomach which was either unresectable or non-curative, and clinically proven as evidenced by involvement of abdominal paraaortic lymph nodes, liver metastasis, or ascites at computed tomography (CT) scan, (2) evaluable lesion on CT scan, at upper gastrointestinal series and upper digestive endoscopy, (3) no prior chemotherapy or radiotherapy, (4) performance status (PS) between 0 and 2 on the Eastern Cooperative Oncology group (ECOG) scale, (5) age less than 75 years, (6) adequate bone marrow function (leukocytes more than $4000 / \mathrm{mm}^{3}$, platelets more than $\left.100000 / \mathrm{mm}^{3}\right)$, (7) adequate liver function (GOT, GPT less than two times the upper limit of normal and total bilirubin less than $2.0 \mathrm{mg} / \mathrm{dl}$ ), (8) adequate renal function (blood urea nitrogen [BUN] and creatinine within normal range), (9) no metastatic tumor to central nervous system, no serious complications (serious infection, heart failure, renal failure, hepatic failure), (10) no concurrent active malignancy, (11) no serious psychosomatic disorder, (12) no second malignancy, and (13) provision of written informed consent. Usual blood chemistry tests and serum carcinoembryonic antigen (CEA) and carbohydrate antigen (CA) 19-9 were performed prior to each cycle. A complete blood and platelet count was done every week to assess hematological toxicity. Pathological specimens were classified as differentiated and undifferentiated carcinoma. We employed neoadjuvant chemotherapy (NAC) with the FLP combination regimen, consisting of 5 -fluorouracil $(5 \mathrm{FU})\left(333 \mathrm{mg} / \mathrm{m}^{2}\right.$ per day intravenous infusion over $2 \mathrm{~h}$ for 8 consecutive days), leucovorin (LV) $(30 \mathrm{mg} /$ body bolus infusion for 8 consecutive days), and cisplatin (CDDP) $\left(50 \mathrm{mg} / \mathrm{m}^{2}\right.$ per day as a 2-h intravenous infusion on days 1 and 8 with hyperhydration) (Table 1). Cycles were repeated every 4 weeks according to hematological, digestive, and renal tolerance. Toxicity was assessed using World Health Organization (WHO) criteria [11]. Patients received at least two cycles of FLP. The evaluation of response was performed immediately after every cycle according to the Japanese Research Society for Gastric Cancer criteria [12]. The duration of treatment was dependent on
Table 1. Protocol schema

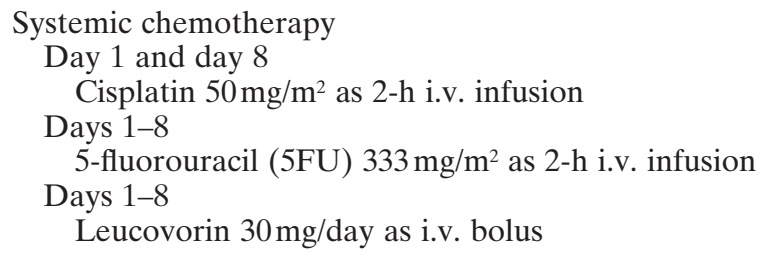

After a waiting period of 3 weeks, repeat at least two cycles

response and toxicity. For patients who achieved an objective response, an additional cycle was planned. Surgery was performed at least 4 weeks after the final cycle. Surgery consisted, when it was possible, of complete excision of tumor and extensive lymphadenectomy. Postoperative additional adjuvant treatment, at least ten cycles of the sequential methotrexate (MTX) and 5FU therapy [13], was performed. If palliative surgery was performed because of incomplete resection, unresectability, or evidence of distant metastases, patients were submitted to another chemotherapy regimen. As a control group, 14 patients with noncurative or unresectable clinical factors who had not received chemotherapy but had had only best supportive care in the same period were compared with the NAC group. A comparison of response rates was performed using the $\chi^{2}$ method. Overall survival was calculated from the start of chemotherapy. Survival comparisons were determined by the log-rank test.

\section{Results}

The patients' characteristics are summarized in Table 2 . As regards the gross type, there were 4 patients with type 2, 9 with type 3 , and 8 with type 4 ; as non-curative factors, there were 5 patients with liver metastasis, 9 with peritoneal dissemination, 17 with involvement of abdominal para-aortic lymph nodes, and 3 with locally advanced and potentially unresectable gastric cancer. All patients were evaluable. The median number of cycles administered per patient was two (range, two to four). The rate of response to chemotherapy was evaluable in 21 patients. There was no complete response (CR), and there were 12 patients with partial response (PR). The overall response rate was $57.1 \%$ (95\% confidence interval [CI], 34.0\%-78.2\%). Eight patients had no change (NC) and there was 1 patient with tumor progression.

Two patients did not undergo surgery because of massive peritoneal carcinosis and para-aortic lymph node enlargement. Thus, 19 patients had an intervention. The primary tumor in $18 / 21$ patients $(85.7 \%)$ 
was resected (12 total gastrectomy and 6 distal gastrectomy). One patient had a pancreatoduodenectomy associated with hepatic central segmentectomy. Another patient with peritoneal carcinomatosis and para-aortic lymph node metastases had a bypass procedure. Of the 21 patients, 33.3\% had a macroscopically complete resection (curative B), 57.1\% had a macroscopically incomplete resection (curative $\mathrm{C}$ ), $4.8 \%$ were unresectable, and $9.5 \%$ were not operated on. Clinical response, toxicity, and survival were assessed.

Table 2. Patients' characteristics

\begin{tabular}{lc}
\hline Characteristics & No. of patients \\
\hline Sex & \\
Male & 14 \\
Female & 7 \\
Age (years) & 57 \\
Mean & $21-75$ \\
Range & \\
Gross type & 4 \\
Type 2 & 9 \\
Type 3 & 8 \\
Type 4 & \\
Histology & 8 \\
Differentiated & 13 \\
Undifferentiated & \\
Surgery & 12 \\
Total gastrectomy & 6 \\
Distal gastrectomy & 3 \\
Unresectable & \\
Unresectable lesions & \\
One factor & 5 \\
N3/4 & \\
Two factors & 2 \\
N3/4,T4 & \\
P3,N4 & 4 \\
H1,N4 & 4 \\
H1,T4 & 1 \\
P3,T4 & 1 \\
Three factors & \\
P2,N4,T4 & \\
H3,P1,T4 & \\
Four factors & \\
H3,P3,N4,T4 & \\
\hline & \\
&
\end{tabular}

Response rate to chemotherapy by tumor site and type

Response rate to chemotherapy according to tumor site and type is shown in Table 3. There was no complete response (CR), but 12 patients $(57.1 \%)$ had a partial response $(\mathrm{PR})$. The response rate was $64.7 \%$ for abdominal para-aortic (No.16) lymph nodes, $47.6 \%$ for primary regions, $40.0 \%$ for liver metastasis, and $11.1 \%$ for peritoneal dissemination. One of 17 patients with No.16 lymph nodes metastases showed complete response of No.16 nodes microscopically and downstaging, but a few other regional lymph nodes revealed remnant cancer cell nests. The response rate according to gross type was $50 \%$ in type $2,66.7 \%$ in type 3 , and $37.5 \%$ in type 4 . Resectability was higher in patients with an objective response to chemotherapy. Of the 12 responders, residual tumor was completely resected in 6. Of the 9 patients who failed to achieve an objective response to chemotherapy, 6 underwent surgery, but complete resection was possible in only 1 of them. Thus, $50 \%$ of the patients with an objective response had a complete resection, compared with $11.1 \%$ of the nonresponders.

\section{Toxicity}

Forty-three cycles were evaluable for toxicity in 21 patients (Table 4). Hematological toxicity consisted of grade 3 leukopenia in 4 patients $(19.0 \%)$ and grade 3 anemia in 3 patients $(14.3 \%)$, but there was no grade 3 thrombocytopenia. Stomatitis grade 3 was noted in 2 patients $(9.5 \%)$ and there was grade 3 hyperbilirubinemia in one patient. There were mild toxicities, such as anorexia, nausea, and diarrhea, but no serious myelosuppression or renal disorders were reported. Therefore, this therapy was well tolerated and there was no increase in postoperative major complications such as anastomotic leakage, adhesive ileus, and/or subphrenic abscess. There was no treatment-related death (TRD) and no grade 4 serious toxicities.

\section{Survival}

With a median follow-up of 35 months (range, 22-46 months), only 3 of the 21 patients $(14.3 \%)$ remain alive

Table 3. Treatment results after FLP $(n=21)$ according to gross type

\begin{tabular}{|c|c|c|c|c|c|}
\hline Gross & Primary & No.16 LN & $\mathrm{H}$ & $\mathrm{P}$ & Total \\
\hline Type 2 & $50.0 \%(2 / 4)$ & $50.0 \%(2 / 4)$ & $0 \%(0 / 1)$ & $0 \%(0 / 1)$ & $50.0 \%(2 / 4)$ \\
\hline Type 3 & $55.6 \%(5 / 9)$ & $71.4 \%(5 / 7)$ & $66.7 \%(2 / 3)$ & $33.3 \%(1 / 3)$ & $66.7 \%(7 / 9)$ \\
\hline Type 4 & $37.5 \%(3 / 8)$ & $66.7 \%(4 / 6)$ & $0 \%(0 / 1)$ & $0 \%(0 / 5)$ & $37.5 \%(3 / 8)$ \\
\hline Total & $47.6 \%(10 / 21)$ & $64.7 \%(11 / 17)$ & $40.0 \%(2 / 5)$ & $11.1 \%(1 / 9)$ & $57.1 \%(12 / 21)$ \\
\hline
\end{tabular}

FLP, 5-Fluorouracil, leukovorin, cisplatin; No.16 LN, Abdominal para-aortic lymph node; H, Liver metastasis; P, peritoneal dissemination 
Table 4. Toxicity ${ }^{\mathrm{a}}$

\begin{tabular}{|c|c|c|c|c|c|c|}
\hline & \multicolumn{4}{|c|}{ Grade } & \multirow[b]{2}{*}{ Positive rate } & \multirow[b]{2}{*}{$\geq$ Grade 3} \\
\hline & 1 & 2 & 3 & 4 & & \\
\hline $\mathrm{Hb}$ & 7 & 10 & 3 & 0 & $20(95.2 \%)$ & $3(14.3 \%)$ \\
\hline WBC & 7 & 5 & 4 & 0 & $16(76.2 \%)$ & $4(19.0 \%)$ \\
\hline Plt & 4 & 1 & 0 & 0 & $5(23.8 \%)$ & $0(0.0 \%)$ \\
\hline $\mathrm{T} \cdot \mathrm{Bil}$ & 0 & 1 & 1 & 0 & $2(9.5 \%)$ & $1(4.8 \%)$ \\
\hline GOT & 3 & 1 & 0 & 0 & $4(19.0 \%)$ & $0(0.0 \%)$ \\
\hline Cre & 1 & 0 & 0 & 0 & $1(4.8 \%)$ & $0(0.0 \%)$ \\
\hline Stomatitis & 8 & 0 & 2 & 0 & $10(42.9 \%)$ & $2(9.5 \%)$ \\
\hline Nausea & 12 & 5 & 0 & 0 & $17(81.0 \%)$ & $0(0.0 \%)$ \\
\hline Diarrhea & 6 & 3 & 0 & 0 & $9(42.9 \%)$ & $0(0.0 \%)$ \\
\hline Alopecia & 3 & 0 & 0 & 0 & $3(14.3 \%)$ & $0(0.0 \%)$ \\
\hline Neurogenic symptoms & 0 & 0 & 0 & 0 & $0(0.0 \%)$ & $0(0.0 \%)$ \\
\hline
\end{tabular}

$\mathrm{Hb}$, Anemia; WBC, leukocytopenia; Plt, thrombocytopenia; TBil, liver dysfunction; GOT, liver dysfunction; Cre, renal dysfunction

a According to WHO criteria [11]

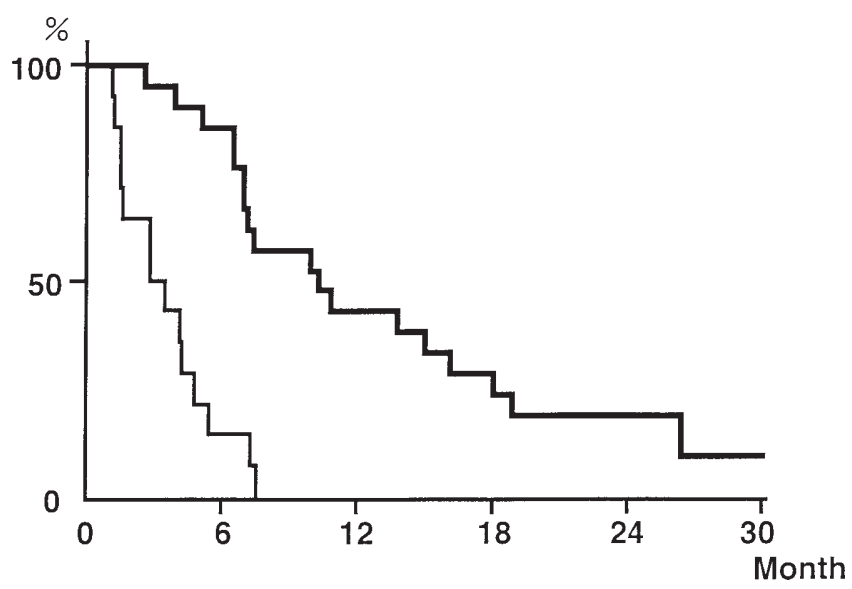

Fig. 1. Actuarial survival curves for 21 gastric cancer patients treated with neoadjuvant chemotherapy (NAC; thick lines) and 14 control patients treated with best supportive care (thin lines). Median survival time (MST) for NAC group, 322 days; MST for controls, 81 days

and disease-free, at $22+, 27+, 32+$ months, respectively, after the beginning of NAC. The median survival time (MST) for the overall population was 322 days and the 1- and 2-year survivals were $42.9 \%$ and $19.0 \%$, respectively (Fig. 1). The MST in the control group was 81 days, and there were no 1-year survivors.

According to the tumor response, the MST of the 12 responders was 571 days, whereas it was 199 days for the 9 non-responders (Fig. 2). The 1- and 2-year survival rates of responders $(66.7 \%$ and $33.3 \%)$ were better than those of the non-responders $(11.1 \%$ and $0 \%)$. There was a significant difference between the two groups $(P<0.01)$.

According to the quality of resection, of the seven patients who had a macroscopically complete resection, four had distant metastases and died (Fig. 3). Survival

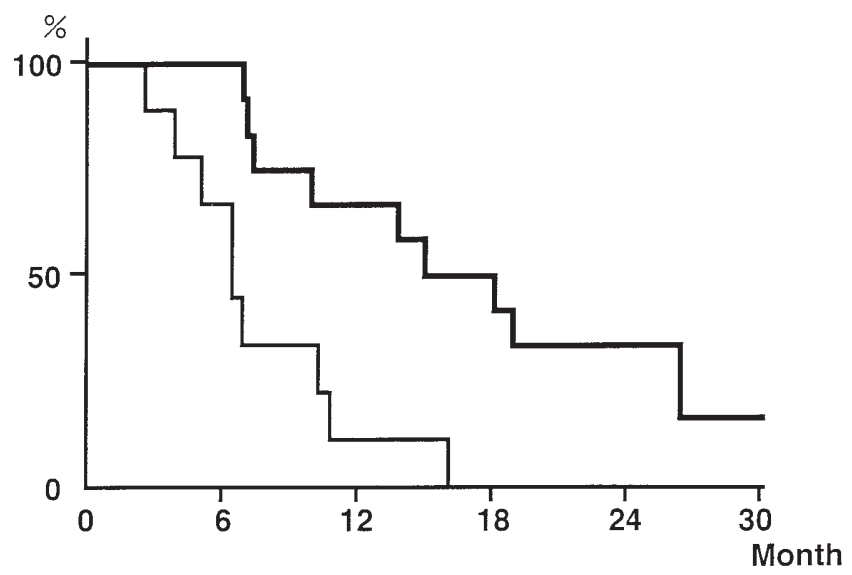

Fig. 2. (thick lines Actuarial survival curves for the 12 responders; MST, 571 days) and 9 non-responders (thin lines; MST, 199 days). Differences between the groups were significant: $P<0.01$ by log-rank test and $P=0.0043$ by generalized Wilcoxon test

was significantly better after curative than after noncurative resection (MST, 835 and 310 days, respectively). The 1- and 2-year survival rates of patients with curative resection were $85.7 \%$ and $57.1 \%$, respectively, with the survival period of the longest survivor, 32 months. The differences between survival rates for the curative and non-curative resection groups were significant $(P<0.01)$.

\section{Discussion}

Japanese studies report favorable results of adjuvant chemotherapy in the limited subsets of patients with advanced gastric cancer [14], but few reports from Western countries support this benefit [15]. With the 


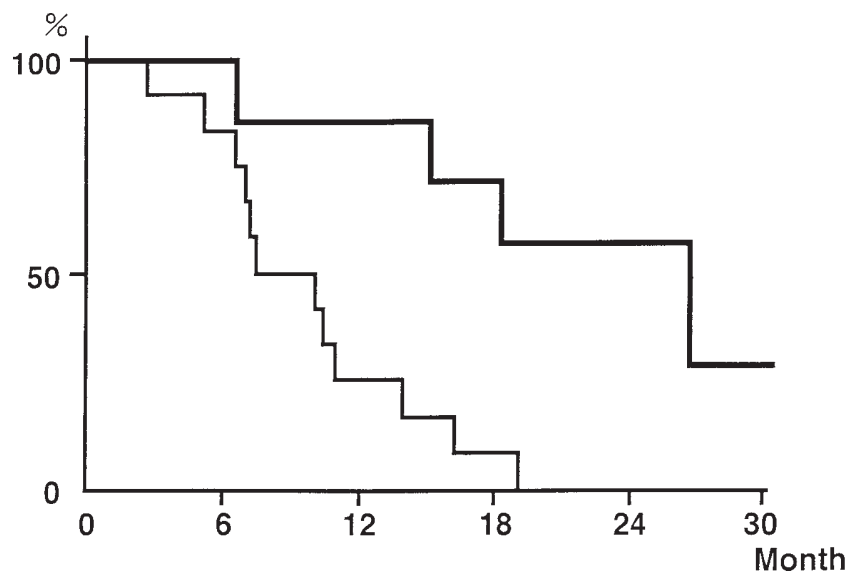

Fig. 3. Actuarial survival curves for 7 patients with curative resection (thick lines; MST, 835 days) and 11 patients with non-curative resection (thin lines; MST, 310 days). Differences between the groups were significant: $P<0.01$ by log-rank test and $P=0.0012$ by generalized Wilcoxon test

development of a chemotherapy regimen that showed stage-dependent activity and predictable response rates, a more systematic assessment of the role of chemotherapy in patients with unresectable or noncurative gastric cancer was possible $[7,16,17]$. The use of NAC in patients with gastric cancer is based on two major premises. One is to reduce the locoregional tumor mass and increase the chance for complete tumor resection on subsequent surgery. The second premise is to eliminate or delay systemic metastases. Both premises are considered essential for prolonging diseasefree and overall survival.

Downstaging of the primary tumor to enable complete resection at subsequent surgery is one of the major goals of NAC. Shrinkage of the primary tumor and para-aortic lymph node and hepatic metastases facilitates resectability. The shrinkage of para-aortic lymph node metastases may be feasible. The response rates of several chemotherapy regimens regarded as effective for advanced gastric cancer are: FLP, 45\% [18]; irinotecan hydrochloride (CPT-11) + cisplatin (CDDP), 41.7\% [19]; FAM (5FU + Adriamycin [ADR] + mitomycin C [MMC]), 42\% [20]; FAP (5FU + ADR + CDDP), 50\% [8]; LV + 5FU, 48\% [21]; MLP-F (methotrexate [MTX] + LV + CDDP + 5FU) 88\% [9], ECF (epirubicin + CDDP + 5FU), 45\% [22]; and FAMTX (5FU + ADR + MTX), 41\% [23]. The response rates of NAC regimens are: EAP (etoposide + $5 \mathrm{FU}+\mathrm{CDDP}$ ) 70\% [6]; EFP (etoposide + 5FU + CDDP), 25\% [24]; FP (CDDP + 5FU) 56\%, [25]; FLEP (etoposide + 5FU + LV + CDDP), 50\% [26]; PMUE $(\mathrm{CDDP}+\mathrm{MMC}+\mathrm{UFT}$ [uracil and futraful] + etoposide) $62 \%$ [16]. The response and curative resection rate were higher in the patients with NAC than in those patients who underwent operation first and re- ceived chemotherapy afterwards [16]. But the prognostic benefit expected from NAC may be limited to a subgroup of patients with locally advanced tumors and distant lymph node metastasis [7,18,25]. The highest response was observed in the metastatic distant lymph nodes (abdominal para-aortic or supraclavicular nodes) and primary lesions [26]. With currently used NAC regimens, response is low for peritoneal carcinosis; our result was $11.1 \%$. Peritoneal dissemination was frequently detected on subsequent resection and was one of the most common reasons precluding complete tumor resection. Because of the poor prognosis associated with peritoneal dissemination and the inefficacy of current neoadjuvant regimens, these patients should not be included in neoadjuvant protocols.

Several arguments have been raised against the more general use of preoperative therapy in patients with advanced gastric tumors. They include the potential toxicity of chemotherapy, the associated deterioration of the general condition of patients while receiving NAC, a delay of definite treatment or tumor progression, and the fear of increased postoperative complications and mortality. Toxicity associated with NAC was reported to be related to treatment-related death (TRD) [27]. EAP was highly effective in locally advanced gastric cancer [6,7], but, on the other hand, TRD of more than $10 \%$ was reported, suggesting that the EAP regimen is too toxic for NAC [28,29]. Myelosuppression with thrombocytopenia and leukocytopenia are the most frequently reported side effects of NAC. Septic complications associated with neutropenia required hospitalization of a substantial number of patients. Myelosuppression could be managed by dose reduction, substitution, or by the use of colony-stimulating factors. It was rare, but chemotherapy-associated deterioration of general status or septic complications prohibited subsequent resection. Close observation is required. Subsequent tumor resection was not performed in $10 \%$ of the patients receiving NAC [30]. Detection of systemic metastases in patients while they were receiving chemotherapy, or peritoneal dissemination detected on laparotomy were the main reasons for not performing a resection. In Japan, the rate of postoperative complications was not increased to the rate observed in patients who had primary resection [18,31]. We experienced a high incidence of toxicity with FP, but its grade was moderate and controllable by symptomatic measures. Ambulatory treatment on an outpatient basis was possible and was safely undertaken [32]. Consequently, the major focus of research clearly must be to develop less toxic and more effective chemotherapy regimens and dosage forms to increase response rates and limit toxicity.

An improvement in overall long-term survival is the ultimate aim of NAC for locally advanced gastric 
cancer. The survival of our patients with PRs with curative surgery was good, but that of PR patients who underwent palliative surgery, and of the non-responders, was poor. In the latter two groups, no survival benefit was observed even when NAC was effective. These results indicate that responsiveness of the tumor and complete surgical eradication seem to enhance longterm survival. The median survival after NAC and subsequent resection ranges between 15 and more than 18 months and is markedly longer than the median survival reported in control groups (6-8 months) [22-24]. Survival after complete tumor resection is another essential reason for assessing the effect of NAC followed by surgery. The median survival after NAC and subsequent complete resection is in the order of 20-25 months. When the survival after NAC and curative resection was compared to that in an age, sex, and tumor stage-matched control group of patients who underwent primary resection, there was a clear tendency indicating a survival advantage for those who had NAC [16]. Furthermore, long-term survivors were exclusively found among patients with distant lymph node metastasis treated by curative surgery, and the 5-year survival rate was surprisingly high (55.6\%) [26]. Disease-free survival and recurrence have usually not been accurately evaluated. Relapse rates after NAC, however, appear to exceed $60 \%$, with a predominance of locoregional recurrences and development of peritoneal metastases. This finding indicates a potential role for postoperative intraperitoneal chemotherapy in addition to preoperative systemic therapy. Postoperative systemic and intraperitoneal therapy, in addition to NAC, may further prolong survival in patients who responded to NAC [14]. Local intraarterial application of NAC is another promising approach that deserves further evaluation [26]. Although NAC may not increase the rate of complete tumor resection in patients with resectable tumors, there appears to be a marked rise in the curative resection rate after NAC in patients with locally advanced tumors. There appears to be a survival benefit in those patients who respond to NAC and who have complete tumor resection at subsequent surgery.

\section{Conclusion}

We concluded that FLP combination therapy for NAC was feasible and useful for primary tumor and abdominal paraaortic metastatic lymph node reduction. A modest survival benefit was observed for responders and for patients who had curative resection. But the survival benefit was not sufficient. We should confirm the effect and survival benefit of FLP for NAC by a prospectively randomized clinical controlled study. Randomized trials assessing the role of preoperative chemotherapy in gastric cancer patients with noncurative clinical factors are, therefore, clearly warranted. Furthermore, more powerful and less toxic regimens are required to improve the results.

\section{References}

1. Maruyama K, Okabayashi K, Kinoshita T. Progress in gastric cancer surgery in Japan and its limits of radicality. World J Surg 1987:11:418-25.

2. Allum WH, Powell DJ, McConkey CC. Gastric cancer: a 25-year review. Br J Surg 1989;76:535-40.

3. Akoh JA, MacIntyre IMC. Improving survival in gastric cancer: review of 5-year survival rates in English language publications from 1970. Br J Surg 1992;79:293-9.

4. Roder JD, Bottcher K, Siewert JR, Busch R, Hermanek P, Meyer HJ. Prognostic factors in gastric cancer: results of the German Gastric Carcinoma group study 1992. Cancer 1993;72:2089-97.

5. Siewert JR, Roder JD, Bottcher K, Busch R, Hermanek P, Meyer HJ. Prognostic relevance of systemic lymph node dissection in gastric carcinoma. Br J Surg 1993;80:1015-8.

6. Wilke H, Preusser U, Gunzer U, Meyer HJ, Siewert JR, Achterrath W, et al. Preoperative chemotherapy in locally advanced and nonresectable gastric cancer; a phase II study with etoposide, doxorubicin, and cisplatin. J Clin Oncol 1989;7:131826.

7. Preusser P, Wilke H, Achterrath W, Fink U, Lenaz L, Heinicke A, et al. Phase II study with combination etoposide, doxorubicin, and cisplatin in advanced measurable gastric cancer. J Clin Oncol 1989;7:1310-17.

8. Moertel CG, Rubin J, O'Connell MJ, Schutt A, Wieand HS. A phase II study of combined 5-fluorouracil, doxorubicin, and cisplatin in the treatment of advanced upper gastrointestinal adenocarcinomas. J Clin Oncol 1986;4:1053-7.

9. Bruckner HW, Cheseer MR, Wong H, Mandeli J. Folate biochemical modulation regimen for the treatment of gastric cancer. J Clin Gastroenterol 1991;13:3884-92.

10. Frei E III. Clinical cancer research: an embattled species. Cancer 1982;50:1979-92.

11. Miller AB, Hoogstraten B, Staquet M, Winkler A. Reporting results of cancer treatment. Cancer 1981;47:207-14.

12. Japanese Research Society for Gastric Cancer. Japanese classification for gastric carcinoma, 1st English ed. 1995; Tokyo: Kanehara.

13. Nashimoto A, Sasaki J. Mitomycin C+ sequential methotrexate and 5-fluorouracil therapy for stage III, IV advanced or recurrent gastric cancers. J Jpn Soc Cancer Ther 1994;29:27-35.

14. Nakajima T. Adjuvant chemotherapy for gastric cancer in Japan: present status and suggestions for rational clinical trials. Jpn J Clin Oncol 1990;20:30-42.

15. Hermans J, Bonenkamp JJ, Boon MC, Bunt AMG, Ohyama S, Sasako M, et al. Adjuvant therapy after curative resection for gastric cancer:meta-analysis of randomized trials. J Clin Oncol 1993;11:1441-7.

16. Yonemura Y, Sawa T, Kinoshita K, Matsuki N, Fushida S, Tanaka S, et al. Neoadjuvant chemotherapy for high-grade advanced gastric cancer. World J Surg 1993:17:256-62.

17. Plukker JTh, Mulder NH, Sleijfer DTh, Grond J, Verschureren RCJ. Chemotherapy and surgery for locally advanced cancer of the cardia and fundus: phase II study with methotrexate and 5fluorouracil. Br J Surg 1991;78:955-8.

18. Leichman L, Silberman H, Leichman CG, Spears CP, Ray M, Muggia FM, et al. Preoperative systemic chemotherapy followed by adjuvant postoperative intraperitoneal therapy for gastric cancer: a University of Southern California pilot program. J Clin Oncol 1992;10:1933-42. 
19. Shirao K, Shimade Y, Kondo H, Saito D, Yamao T, Ono H, et al. Phase I-II study of irinotecan hydrochloride combined with cisplatin in patients with advanced gastric cancer. J Clin Oncol 1997;15:921-7.

20. Mac Donald JS, Schein PS, Wolley PV, Smythe T, Ueno W, Hoth $\mathrm{D}$, et al. 5-Fluorouracil, doxorubicin and mitomycin C (FAM) combination chemotherapy for advanced gastric cancer. Ann Intern Med 1980;93:533-6.

21. Machover D, Goldschmidt E, Chollet P, Metzger G, Zittoun J, Marquet J, et al. Treatment of advanced colorectal and gastric adenocarcinomas with 5-fluorouracil and high-dose folinic acid. J Clin Oncol 1986;4:685-96.

22. Webb A, Cunningham D, Scarffe JH, Harper P, Norman A, Joffe JK, et al. Epirubicin, cisplatin and fluorouracil versus fluorouracil, doxorubicin and methotrexate. J Clin Oncol 1997;15: 261-7.

23. Wils JA, Klein HO, Wagener DJ, Bleiberg H, Reis H, Korsten F, et al. for EORTC. Sequential high-dose methotrexate and fluorouracil combined with doxorubicin. A step ahead in the treatment of advanced gastric cancer: a trial of the EORTC gastrointestinal tract Cooperative Group. J Clin Oncol 1991;9:82731.

24. Ajani JA, Ota DM, Jessup M, Ames FC, McBride C, Boddie A, et al. Resectable gastric carcinoma-an evaluation of preoperative and postoperative chemotherapy. Cancer 1991;68:1501-6.

25. Rougier Ph, Mahjoubi M, Lesser Ph, Ducreux M, Oliveira J, Ychou M, et al. Neoadjuvant chemotherapy in locally advanced gastric carcinoma-a phase II trial with combined continuous intravenous 5-fluorouracil and bolus cisplatinum. Eur J Cancer 1994;30:1269-75.

26. Nakajima T, Ota K, Ishihara S, Oyama S, Nishi M, Ohashi Y, et al. Combined intensive chemotherapy and radical surgery for incurable gastric cancer. Ann Surg Oncol 1997;4:203-8.

27. Kelsen D, Karpeh M, Schwartz G, Gerdes H, Lightdale C, Botet $\mathrm{J}$, et al. Neoadjuvant therapy of high-risk gastric cancer: a phase II trial of preoperative FAMTX and postoperative intraperitoneal fluorouracil-cisplatin plus intravenous fluorouracil. J Clin Oncol 1996;14:1818-28.

28. Lerner A, Gonin R, Steele GD, Mayer RJ. Etoposide, doxorubicin, and cisplatin chemotherapy for advanced gastric adenocarcinoma. Results of a phase II trial. J Clin Oncol 1992;10:536-40.

29. Kelsen D, Atiq OT, Saltz L, Niedzwiecki D, Ginn D, Chapman D, et al. FAMTX versus etoposide, adriamycin and cisplatin: a randomized assignment trial in gastric cancer. J Clin Oncol 1992;10:541-8.

30. Fink U, Schuhmacher C, Stein HJ, Busch R, Feussner H, Dittler $\mathrm{HJ}$, et al. Preoperative chemotherapy for stage III-IV gastric carcinoma: feasibility, response and outcome after complete resection. Br J Surg 1995;82:1248-52.

31. Nashimoto A, Sasaki J, Tanaka O, Tsutsui M, Tsuchiya Y, Makino H. Neoadjuvant chemotherapy for advanced gastric carcinoma with non-curative clinical factors. Jpn J Gastroenterol Surg 1998;31:2199-205.

32. Nashimoto A, Sasaki J, Akai S. Ambulatory combination chemotherapy with cisplatin and 5-fluorouracil for advanced gastric cancer patients. J Jpn Soc Cancer Ther 1992;27:654-64. 